\title{
Relativistic Doppler effect: universal spectra and zeptosecond pulses.
}

\author{
S. Gordienko ${ }^{1,2}$, A. Pukhov ${ }^{1}$, O. Shorokhov ${ }^{1}$, and T. Baeva ${ }^{1}$ \\ ${ }^{1}$ Institut für Theoretische Physik I, Heinrich-Heine-Universität Düsseldorf, D-40225, Germany \\ ${ }^{2}$ L. D. Landau Institute for Theoretical Physics, Moscow, Russia
}

(Dated: October 28, 2018)

\begin{abstract}
We report on a numerical observation of the train of zeptosecond pulses produced by reflection of a relativistically intense femtosecond laser pulse from the oscillating boundary of an overdense plasma because of the Doppler effect. These pulses promise to become a unique experimental and technological tool since their length is of the order of the Bohr radius and the intensity is extremely high $\propto 10^{19} \mathrm{~W} / \mathrm{cm}^{2}$. We present the physical mechanism, analytical theory, and direct particle-incell simulations. We show that the harmonic spectrum is universal: the intensity of $n$th harmonic scales as $1 / n^{p}$ for $n<4 \gamma^{2}$, where $\gamma$ is the largest $\gamma$-factor of the electron fluid boundary, $p=3$ and $p=5 / 2$ for the broadband and quasimonochromatic laser pulses respectively.

PACS numbers: 03.30+p, 03.50.De, 42.65.Re, 42.65.Ky
\end{abstract}

Recent impressive progress in the physics of attosecond X-ray pulses [1] triggers a fascinating question whether a range of even shorter pulses is achievable with the contemporary or just coming experimental technology. In the present work, we show that when an ultrarelativistically intense laser pulse, $I \lambda_{0}^{2} \geq 10^{20} \mathrm{~W} / \mathrm{cm}^{-2}$ $\mu m^{2}$, is incident onto a plasma-vacuum boundary, the reflected radiation naturally contains subattosecond, or zeptosecond (zepto $=10^{-21}$ ) pulses.

The idea of harmonic generation via reflection from a laser-driven oscillating plasma boundary was first proposed a few years ago and has been studied in several theoretical articles [2, 3, 4]. Numerous experimental works [5, [6, 7] and simulations [8, 9] were devoted to the laser interactions with overdense plasmas and the high-harmonic generation. Plaja et al [3] were the first to realize that the simple concept of an oscillating plasma mirror gives an opportunity to produce extremely short pulses. In a recent work, Naumova et al $\underline{\underline{4}}]$ have made a revolutionary step in this direction since they have proven that a single attosecond pulse can be isolated when a laser pulse focused down to the $\lambda^{3}$ volume is reflected from a plasma surface.

The emphasis of our work is different. We concentrate on the very basic physics of the high harmonic generation and discover the spectrum universality as well as its scaling. These features have been overseen in the earlier publications. The central result of our present work is the universal power-law harmonics spectrum decaying as $1 / n^{p}$. The exponent $p=5 / 2$ for a quasi-monocromatic and $p=3$ for a broad band incident laser pulse. This power-law spectrum runs up to a critical harmonic number $n_{c} \propto 4 \gamma_{\text {max }}^{2}$, where an exponential cut-off sets in. Here $\gamma_{\max }$ is the largest relativistic factor of the plasma boundary. It is the slow power-law decay, that allows for the subattosecond pulses. We compare the analytic theory with direct particle-in-cell (PIC) simulations.

Let us consider a monochromatic laser wave with the dimensionless vector potential $e \mathbf{A}_{i}(t, x) / m c^{2}=$ $\operatorname{Re}\left[\mathbf{a}_{0} \exp (-i \omega t-i \omega x / c)\right]$. This wave is reflected by a sharp plasma surface positioned at $X\left(t^{\prime}\right)$ at the time $t^{\prime}$.
First of all, we must decide what boundary conditions should be used. We are interested in reflection from the overdense plasma $N_{e} \gg N_{c}$ with $\Gamma=a_{0}\left(N_{c} / N_{e}\right) \ll 1$, where $N_{e}$ is the plasma electron density, and $N_{c}=$ $\omega^{2} m_{e} / 4 \pi e^{2}$ is the critical density.

The "ideal mirror" boundary condition implies zero tangential components of the vector potential at the mirror surface. As a consequence, when the ideal mirror moves with $\gamma \gg 1$ toward a laser pulse with the electric field $E_{l}$ and duration $\tau$, then the reflected pulse acquires the electric field $E_{\text {refl }} \propto \gamma^{2} E_{l}$ and the duration $\tau_{\text {refl }} \propto \tau / \gamma^{2}$. Consequently, the energy of the reflected pulse must be $\gamma^{2}$ times higher than that of the incident one. However, as the plasma surface is driven by the same laser pulse, this scaling is energetically prohibited, and the plasma cannot serve as an "ideal mirror". Indeed, the ideal mirror must support a surface current $J_{m} \propto e N_{c} \gamma a c^{2} / \omega$ growing with the $\gamma$-factor. A realistic plasma surface does not provide such a current and the boundary condition must be changed.

Let us consider the tangential vector potential component of a laser pulse normally incident onto an overdense plasma slab:

$$
\frac{1}{c^{2}} \frac{\partial^{2} \mathbf{A}(t, x)}{\partial t^{2}}-\frac{\partial^{2} \mathbf{A}(t, x)}{\partial x^{2}}=\frac{4 \pi}{c} \mathbf{j}(t, x),
$$

where $\mathbf{A}(t, x=-\infty)=0$ and $\mathbf{j}$ is the tangential component of plasma current density. Eq. (11) yields

$$
\mathbf{A}(t, x)=2 \pi \int_{-\infty}^{+\infty} \mathbf{J}\left(t, x, t^{\prime}, x^{\prime}\right) d t^{\prime} d x^{\prime} .
$$

Here $\mathbf{J}\left(t, x, t^{\prime}, x^{\prime}\right)=\mathbf{j}\left(t^{\prime}, x^{\prime}\right)\left(\Theta_{-}-\Theta_{+}\right)$. We have defined $\Theta_{-}=\Theta\left(t-t^{\prime}-\left|x-x^{\prime}\right| / c\right)$ and $\Theta_{+}=$ $\Theta\left(t-t^{\prime}+\left(x-x^{\prime}\right) / c\right), \quad \Theta(t)$ is the Heaviside stepfunction. Due to this choice of $\mathbf{J}$ the vector potential $\mathbf{A}(t, x)$ satisfies both Eq. (1) and the boundary condition at $x=-\infty$ since $\mathbf{J}\left(t, x=-\infty, t^{\prime}, x^{\prime}\right)=0$. The 


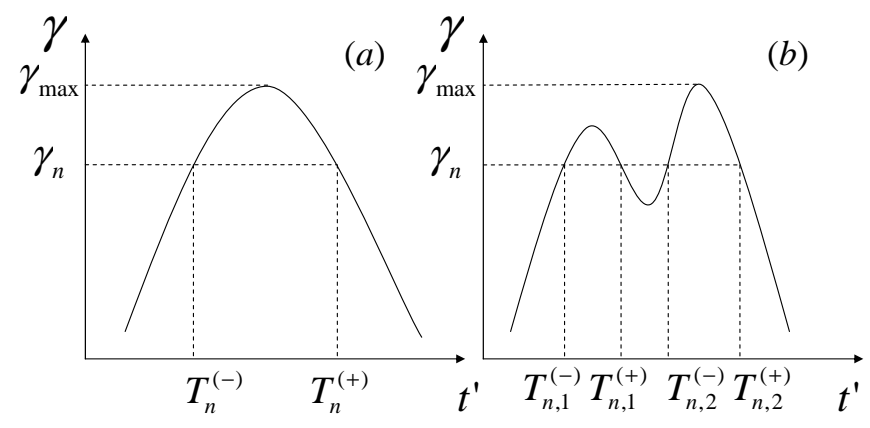

FIG. 1: A sketch of possible motions of the plasma surface. (a) Single "hump" per half-laser period: two saddle points are responsible for the generation of $n$th harmonic. (b) More complex plasma surface motion, the saddle points appear in pairs, two per "hump". Interference between the different saddle points leads to modulations of the spectrum [7].

tangential electric field is $\mathbf{E}_{t}=-(1 / c) \partial_{t} \mathbf{A}(t, x)$. At the electron fluid surface $X(t)$ we have

$$
\mathbf{E}_{t}(t, X(t))=\frac{2 \pi}{c} \sum_{\alpha=-1}^{\alpha=+1} \alpha \int_{0}^{-\infty} \mathbf{j}(t+\alpha \xi / c, X(t)+\xi) d \xi
$$

where $\xi=x^{\prime}-X(t)$. If the characteristic time $\tau$ of the skin layer evolution is long in the sense $c \tau \gg \delta$, where $\delta$ is the plasma skin length, then we can Taylorexpand: $\mathbf{j}\left(t \pm \xi / c, x^{\prime}=X(t)+\xi\right) \approx \mathbf{j}\left(t, x^{\prime}\right) \pm \epsilon$, where $\epsilon=(\xi / c) \partial_{t} \mathbf{j}\left(t, x^{\prime}\right)$. We substitute this expression into (3). The zero order terms cancel one another and we get $\mathbf{E}_{t}(t, X(t)) \propto J_{p}(\delta / c \tau) \ll E_{l}$, where $J_{p} \propto c E_{l}$ is the maximum plasma surface current. Thus, as long as the skin-layer is thin and the plasma surface current is limited, we can use the Leontovich boundary condition [10]

$$
\mathbf{E}_{t}(t, X(t))=0
$$

The same boundary condition was postulated ad hoc by Plaja et al [3] to interpret their PIC simulation results. In the present work, we substantiate the boundary condition (4) physically. Using it we are able to derive very general properties of the reflected radiation.

According to Eq. (4), the reflected wave electric field at the plasma surface is $\mathbf{E}_{r}\left(t^{\prime}, X\left(t^{\prime}\right)\right)=-\mathbf{E}_{i}\left(t^{\prime}, X\left(t^{\prime}\right)\right)$, where $\mathbf{E}_{i}\left(t^{\prime}, X\left(t^{\prime}\right)\right)=-(1 / c) \partial_{t^{\prime}} \mathbf{A}_{i}\left(t^{\prime}, X\left(t^{\prime}\right)\right)$ is the incident laser field, $t^{\prime}$ is the reflection time. The onedimensional (1d) wave equation simply translates a signal in vacuum. Thus the reflected wave field at the observer position $x$ and time $t$ is $\mathbf{E}_{r}(t, x)=-\mathbf{E}_{i}\left(t^{\prime}, X\left(t^{\prime}\right)\right)$. Setting $x=0$ at the observer position we find that the Fourier spectrum of the electric field $\mathbf{E}_{r}(t, x=0)$ coincides with the spectrum of $F(t)=\left(A_{0} \omega / c\right) \cos \left(2 \omega t^{\prime}-\omega t\right)$, where

$$
t^{\prime}-X\left(t^{\prime}\right) / c=t
$$

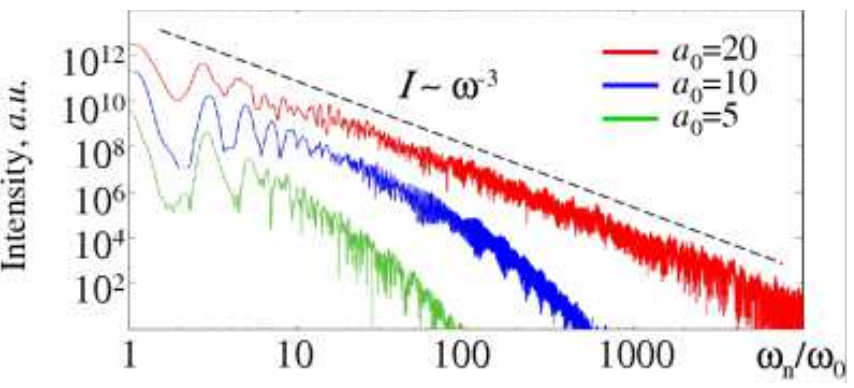

FIG. 2: Spectra of the reflected radiation for the laser amplitudes $a_{0}=5,10,20$. The broken line marks the universal scaling $I \propto \omega^{-3}$.

is the retardation relation. The fine structure of the spectrum of $F(t)$ depends on a particular surface motion $X(t)$, which is defined by complex laser-plasma interactions at the plasma surface. The previous theoretical works on high order harmonic generation from plasma surfaces [2, 3] tried to approximate the function $X(t)$.

It appears, however, that a universal spectrum scaling can be obtained without an exact knowledge of the function $X(t)$. Being interested in universal results we discard calculating $X(t)$ and this makes our approach very different from the previous ones. We only suppose for a moment that the boundary motion is periodic $X(t+\pi / \omega)=X(t)$. Later we consider nonmonochromatic laser pulses and get rid even of this restriction. First, we mention that the Fourier spectrum of $F(t)$ can be represented in the form:

$$
\hat{F}_{n}=A_{0} \omega\left[\hat{C}_{n+1}+\hat{C}_{n-1}-i\left(\hat{S}_{n+1}-\hat{S}_{n-1}\right)\right] /(2 c),
$$

where $\hat{C}_{n}$ and $\hat{S}_{n}$ are the $n$-th harmonics of $C(t)=$ $\cos \left(2 \omega t^{\prime}\right)$ and $S(t)=\sin \left(2 \omega t^{\prime}\right)$, and $t^{\prime}$ is the retarded time (5). We examine only the spectrum of $C(t)$, because that of $S(t)$ can be worked out analogously.

It is easy to see that the function $C(t)$ has a period $\pi / \omega$. Thus, its spectrum contains only even laser harmonics. Introducing the new variable $\tau=2 t$ we obtain

$$
\hat{C}_{n=2 m}=\frac{1}{2} \int_{-\pi / 2 \omega}^{\pi / 2 \omega}\left[\exp \left(i \Phi_{1}(\tau)\right)+\exp \left(i \Phi_{2}(\tau)\right)\right] d \tau
$$

where $\Phi_{1}(\tau)=(1-m) \omega \tau+2 \Phi_{r}(\tau), \Phi_{2}(\tau)=-(1+m) \omega \tau-$ $2 \Phi_{r}(\tau)$, and $\Phi_{r}(\tau)=(\omega / c) X(\arccos C(\tau / 2) /(2 \omega))$ is the retarded phase. The definition of $\Phi_{r}(\tau)$ is recurrent, because $C(t)$ itself is defined through $X$.

To calculate the spectrum (17) we use the saddle point technique. The saddle points $\tau_{n}$ are obtained from the equations $d \Phi_{1}\left(\tau_{n}\right) / d \tau=0$ and $d \Phi_{2}\left(\tau_{n}\right) / d \tau=0$. The first equation reduces to $d \Phi_{r}\left(\tau_{n}\right) / d \tau=n / 2-1$. Taking the first derivative of the recurrent definition of $\Phi_{r}$ we 
re-write the saddle-point equation as

$$
d X\left(T_{n}\right) / d T=c(1-2 / n) \text { or } n \approx 4 \gamma^{2}\left(T_{n}\right),
$$

where $T(\tau)=(1 / 2 \omega) \arccos C(\tau / 2), T_{n}=T\left(\tau_{n}\right)$ and $\gamma=1 / \sqrt{1-\beta^{2}}, c \beta=d X(T) / d T$. Eq. (8) has a clear physical meaning. The reflected radiation frequency is multiplied by the factor $4 \gamma^{2}$ because of the relativistic Doppler effect, where $\gamma$ is the relativistic factor of the plasma surface. If the plasma surface oscillates nonrelativistically, so that $\gamma \approx 1$, then Eq. (8) has no real solutions for $n>1$, and the spectrum of $C(t)$ exponentially decays. When $\gamma \gg 1$, there is a real solution for any $n<n_{c}=4 \gamma_{\max }^{2}$. A similar consideration shows that the second saddle-point equation has no real solutions. The spectrum is thus

$$
\hat{C}_{n}=\sum_{\tau_{n}} \sqrt{2 \pi /\left|d_{t}^{2} \Phi_{r}\left(\tau_{n}\right)\right|} \exp \left(i \Phi_{1}\left(\tau_{n}\right) \pm i \pi / 4\right)
$$

To estimate (9) we use the fact that the highest harmonics are generated around the time $T_{\max }$, when the plasma surface moves toward the laser with the highest relativistic factor $\gamma_{\max }$. In its vicinity, one can approximate $\gamma \approx \gamma_{\max }\left[1-g^{2}\left(T-T_{\max }\right)^{2} / 2\right]$ as shown in Fig. 1]. A straightforward algebra leads to

$$
\left|d_{t}^{2} \Phi_{r}\left(\tau_{n}\right)\right|=D g \omega n^{2} \sqrt{n\left(n_{c}-n\right) / n_{c}}+O\left(n^{2}\right),
$$

where $D=\sin \left(2 \omega T_{\max }\right) / 2 \gamma_{\max }$. The estimate (10) leads to the spectrum intensity scaling

$$
\hat{C}_{n}^{2} \propto n^{-5 / 2} \quad \text { for } \quad n<n_{c}
$$

When one considers the physical mechanism of highharmonic generation as presented in Fig. 1] it becomes evident that the harmonics are emitted in the form of subattosecond pulses. Indeed, all harmonics above a number $n<n_{c}$ are generated at times between $T_{n}^{(-)}$ and $T_{n}^{(+)}$. From Eqs. (5), (8) the subattosecond pulse duration $\Delta \tau=t\left(T_{n}^{(+)}\right)-t\left(T_{n}^{(-)}\right)$as it is seen by the observer is $\Delta \tau=(4 / 3 g n) \sqrt{n_{c} / n}$ for $1 \ll n \ll n_{c}$. This estimation tells us that the reflected pulse can be made very short by applying a filter selecting harmonics with high numbers larger than $n$.

The mechanism presented in Fig. 11 has another very interesting consequence. Each harmonic is generated due to the saddle points corresponding to the proper $\gamma-$ factor. These saddle points come into (9) with different phase multipliers. Fig. 1b represents the case of a more complicated plasma surface motion, when the $\gamma-$ factor has several maxima, "humps", per half-laser period. Yet, one can see that the saddle points are grouped, i.e. there is a couple of the saddle points on every "hump".

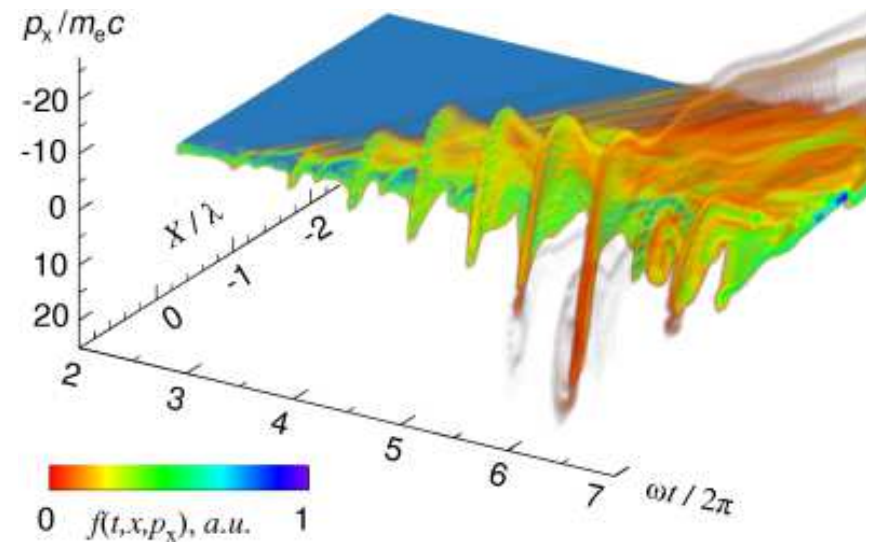

FIG. 3: Electron distribution function The helix represents the electron surface motion in the laser field. The reddish downward spikes stay for the surface relativistic motion towards the laser. These spikes are responsible for the zeptosecond pulse generation.

The phase shift between the contributions of the saddle points $T_{n, i}^{( \pm)}$belonging to the the same $i$ th "hump" is not very large. For example, for the saddle points $T_{n}^{( \pm)}$ this phase shift equals $\Phi(n)=-(5 \omega / 3 g) \sqrt{n_{c} / n}$. The frequency modulation period due to their interference is $\hat{\Omega} \propto 2 \pi \omega /(d \Phi(n) / d n)$, i.e. $\hat{\Omega} \propto\left(6 \pi g / 5 \sqrt{n_{c}}\right) n^{3 / 2}$. This inteference really brings modulation to the spectrum only if $\sqrt{n_{c}} \gg 6 \pi g /(5 \omega)$. On the other side, the phase shift between the contributions from different "humps" can be much larger. This means that a non-trivial motion of the critical surface producing more than one $\gamma$-factor "hump" per oscillation period is the cause of the spectrum modulation. This conclusion supports the explanation of the modulation proposed by Watts et al [7] and agrees with the experimental observations by Teubner et al [6] and Watts et al 7]. Finally, we notice that the larger number of the saddle points does not change the averaged value for $d_{t}^{2} \Phi\left(t_{n}\right)$ and, consequently, does not affect the overall spectrum scaling.

A careful analysis reveals that the intensity spectrum $\propto 1 / n^{5 / 2}$ is valid for a monochromatic incident wave only. Indeed, if the laser pulse is short and has a spectral bandwidth $\Delta \omega$, then the spectral scaling fails at frequencies $\Omega>\omega^{2} / \Delta \omega$. At these frequencies, the reflected radiation is broadband and contains no distinguishable harmonics. To examine the finite bandwidth influence we represent the incident wave as $\mathbf{E}_{i}(t, x)=$ $\operatorname{Re}\left[\omega \mathbf{A}_{0} \exp (-i \phi(t+x / c))\right]$, where $\partial_{t} \phi(t+x / c) \approx \omega$ and $\Delta \omega \ll \omega$. A procedure almost identical to that leading to Eqs. (7) and (9) reduces the Fourier spectrum calculation of $\mathbf{E}_{r}$ to that of $C(t)=\exp (-i \phi(T(t)))$, where $T(t)=-t+2 X\left(t^{\prime}\right) / c, 2 t^{\prime}=t+F(i \ln C(t))$ and $F(\phi(t))=t$ for any $t$.

To apply the steepest descent method to evaluate the $\Omega$-Fourier amplitude $\hat{C}_{\Omega}$ we introduce a new function $\Phi=\operatorname{Re}(\phi)$ such that $d_{t} \Phi\left(-t+2 X\left(t^{\prime}\right) / c\right)=\Omega$. We have neglected $\operatorname{Im}(\phi)$ because the shift of the saddle point due 


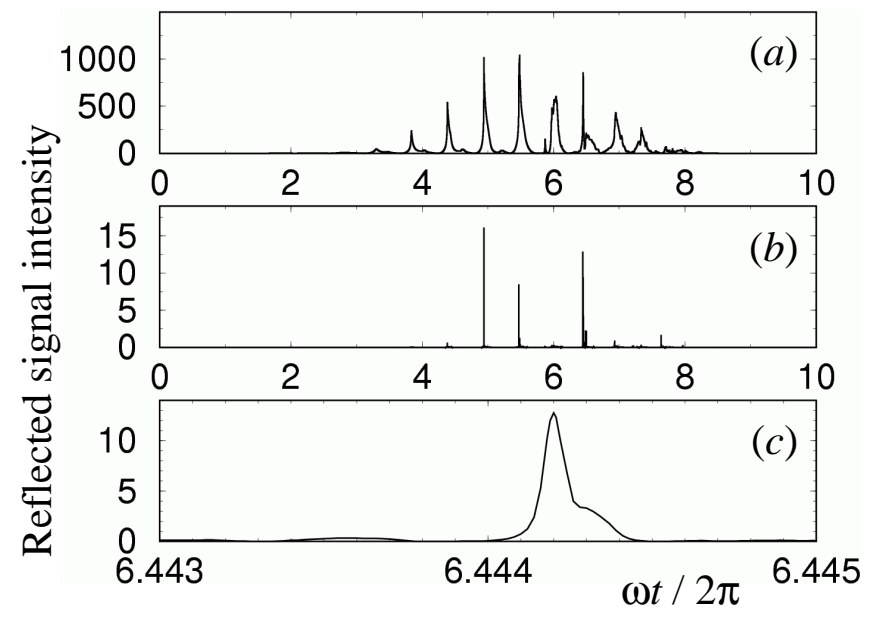

FIG. 4: Zeptosecond pulse train: a) temporal structure of the reflected radiation; b) zeptosecond pulse train seen after spectral filtering; c) one of the zeptosecond pulses zoomed, its FWHM duration is about $300 \mathrm{zs}$.

to $\operatorname{Im}(\phi)$ is only $O\left((\omega / \Omega)^{2}\right)$. Instead of the monochromatic expression (9) we obtain now $\hat{C}_{\Omega} \propto 1 / \sqrt{\left|d_{t}^{2} \Phi\right|}$, where

$$
d_{t}^{2} \Phi=\left(\frac{\Omega}{\omega}\right)^{3}\left(B+O\left(\frac{g \omega}{\gamma_{\max }} \sqrt{\frac{\left(2 \omega n_{c}-\Omega\right)}{n_{c} \Omega}}\right)\right)
$$

for $\Omega<2 \omega n_{c}$. Here, the $O(\ldots)$-term on the right hand part of Eq.(12) is inherited from the monochromatic approximation, see Eq. (10). The new term $B=$ $\left|2 \omega C_{\max } d / d T\left[\left(d \Phi\left(T_{\max }\right) / d T\right) /\left(d \phi\left(T_{\max }\right) / d T\right)\right]\right|$ appears due to the finite spectral width. Here $T_{\max }, n_{c}$ and $g$ are the same as in Eq. (10), and $C_{\max }=C\left(T_{\max }\right)$.

One readily finds that $B \propto 1 / \tau^{2}$, where $\tau \propto 1 / \Delta \omega$ is the incident pulse duration. Thus, we can neglect the $O(\ldots)$-term for $\Omega / \omega>n_{c 1}=(g \omega) /\left(\gamma_{\max }^{2} B^{2}\right)$ what leads to

$$
I_{\Omega} \propto\left|\hat{C}_{\Omega}\right|^{2} \propto 1 / \Omega^{3}, \quad \Omega=n \omega
$$

We emphasize that the criterion $\Omega>n_{c 1}$ defining the transition from $1 / \omega^{5 / 2}$-spectrum to $1 / \omega^{3}$-spectrum depends on the function $B$ that will be analised in detail elsewhere. Altogether, the spectral intensity $I_{n}$ of the reflected radiation scales as $I_{\Omega} \propto 1 / n^{5 / 2}$ for $1<n<n_{c 1}$, then as $I_{\Omega} \propto 1 / n^{3}$ for $n_{c 1}<n<4 \gamma_{\max }^{2}$ and finally decays exponentially for $n>4 \gamma_{\max }^{2}$.
To check our analytical results, we have done a number of 1d PIC simulations. A laser pulse with the Gaussian profile $a=a_{0} \exp \left(-t^{2} / \tau_{L}^{2}\right)$ was incident onto a plasma layer with a step density profile. Fig. 2 shows spectra of the reflected radiation for laser amplitudes $a_{0}=5,10,20$, duration $\omega \tau_{L}=4 \pi$ and the plasma density $N_{e}=30 N_{c}$, which roughly corresponds to the solid hydrogen or liquid helium. The log-log scale of Fig. [2] reveals the power-law scaling of the spectral intensity $I_{\Omega} \propto 1 / n^{3}$. The critical harmonic number $n_{c}$, where the power-law scaling changes into the exponential decay increases for higher laser amplitudes. Also, the spectral intensity modulations are seen [6, 7].

Let us take a close look at the particular case $a_{0}=20$ (the red line in Fig. (2). In this case, the power-law spectrum extends above the harmonic number 2000, and zeptosecond pulses can be generated. As one sees from the electron distribution function $f\left(t, x, p_{x}\right)$, Fig. 3] the maximum surface $\gamma$-factor $\gamma_{\max } \approx 25$ is achieved at the time $t \approx 6$. The temporal profile of the reflected radiation is shown in Fig. [4 When no spectral filter is applied, Fig. [a, a train of attosecond pulses is observed [3]. However, when we apply a spectral filter selecting harmonics above $n=300$, a train of much shorter pulses is recov-

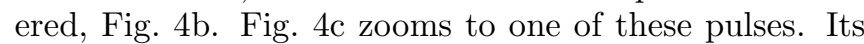
full width at half maximum is about $300 \mathrm{zs}$. At the same time its intensity normalized to the laser frequency is huge $\left(e E_{z s} / m c \omega\right)^{2} \approx 14$ that would correspond to the intensity $I_{z s} \approx 2 \times 10^{19} \mathrm{~W} / \mathrm{cm}^{2}$.

The presented theory and simulations are $1 \mathrm{~d}$, i.e., in a planar geometry. This assumes that the laser focal spot radius $r \gg \lambda$. Recent $2 \mathrm{~d}$ and $3 \mathrm{~d}$ PIC simulations in the $\lambda^{3}$ regime [4] have shown that the multi-dimensional effects may help, particularly, to isolate a single (sub)attosecond pulse.

In conclusion, the reflection from ultrarelativistically moving plasma boundaries forms two different universal spectra because of the relativistic Doppler effect: $1 / n^{5 / 2}$ for an incident quasimonochromatic wave and $1 / n^{3}$ for a broadband pulse. The slow power law decay of the high harmonic spectrum causes high intensity zeptosecond pulses. The observed pulse duration of 300 zs corresponds to the spatial pulse extent comparable to the first Bohr orbit. As a result, the special relativity theory introdicing the relativistic Doppler effect may open a new page of applied ultrafast laser spectroscopy.

We gratefully acknowledge discussions with Prof. J. Meyer-ter-Vehn and Dr. G. D. Tsakiris. The work has been supported by RFFI 04-02-16972, the AvH fund and DFG.
[1] F. Krausz, Phys. World 14 (9), 41 (2001); M. Lewenstein, Science 297, 1131 (2002); Ph. Bucksbaum Nature 421, 593 (2003); N. A. Papadogiannis, et al, Phys. Rev. Lett.
83, 4289 (1999); A. Pukhov, S. Gordienko, T. Baeva, Phys. Rev. Lett. 91, 173002 (2003).

[2] R. Lichters et al, Phys. Plasmas 3, 3425 (1996); D. von 
der Linde and K. Rzazewski, Appl. Phys. B: Lasers Opt. 63499 (1996).

[3] L. Plaja, L. Roso, K. Rzazewski, M. Lewenstein, J. Opt. Soc. Am. B 7, 1904 (1998).

[4] N. M. Naumova, J. A. Nees, I. V. Sokolov, B. Hou, and G. A. Mourou, Phys. Rev. Lett. 92, 063902 (2004).

[5] S. Kohlweyer et al, Opt. Commun. 117, 431 (1995); P. A. Norreys et al, Phys. Rev. Lett. 76, 1832 (1996).

[6] U. Teubner, G. Pretzler, Th. Schlegel et al, Phys. Rev. A, 013816 (2003).
[7] I. Watts, et al, Phys. Rev. Lett. 88, 155001-1 (2002).

[8] P. Gibbon, Phys. Rev. Lett. 7650 (1996).

[9] S. C. Wilks et al, Phys. Rev. Lett. 69, 1383 (1992); H. Ruhl and P. Mulser, Phys. Lett. A 205388 (1995); P. Gibbon and A. R. Bell, Phys. Rev. Lett. 68 (1992).

[10] L. D. Landau, E. M. Lifshitz, and L. P. Pitaevskii, Electrodynamics of Continuous Media, Pergamon Press, Oxford, 1984. 